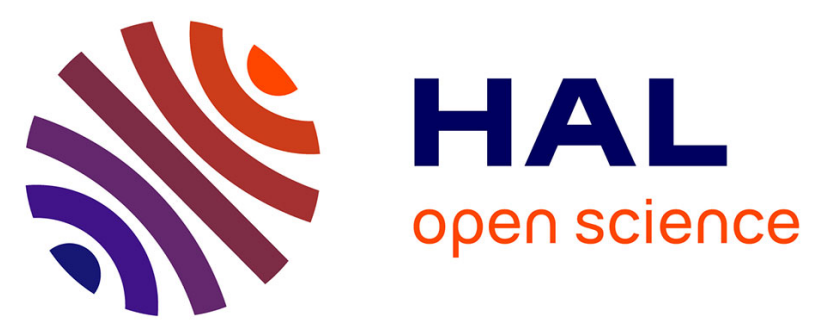

\title{
Dynamic Sensitivity and Noise Floor of a Bonded Magneto(Elasto)Electric Laminate for Low Frequency Magnetic Field Sensing under Strain Modulations
}

Xin Zhuang, Marc Lam Chok Sing, Christophe Dolabdjian, Yanping Wang, Peter Finkel, Jiefang Li, Dwight Viehland

\section{To cite this version:}

Xin Zhuang, Marc Lam Chok Sing, Christophe Dolabdjian, Yanping Wang, Peter Finkel, et al.. Dynamic Sensitivity and Noise Floor of a Bonded Magneto(Elasto)Electric Laminate for Low Frequency Magnetic Field Sensing under Strain Modulations. Key Engineering Materials, 2015, 644, pp.236-239. 10.4028/www.scientific.net/KEM.644.236 . hal-01162289

\section{HAL Id: hal-01162289 \\ https://hal.science/hal-01162289}

Submitted on 10 Jun 2015

HAL is a multi-disciplinary open access archive for the deposit and dissemination of scientific research documents, whether they are published or not. The documents may come from teaching and research institutions in France or abroad, or from public or private research centers.
L'archive ouverte pluridisciplinaire HAL, est destinée au dépôt et à la diffusion de documents scientifiques de niveau recherche, publiés ou non, émanant des établissements d'enseignement et de recherche français ou étrangers, des laboratoires publics ou privés. 


\title{
Dynamic Sensitivity and Noise Floor of a Bonded Magneto(elasto)Electric Laminate for Low Frequency Magnetic Field Sensing under strain modulations
}

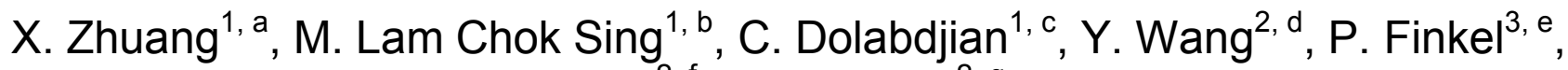 \\ J. Li ${ }^{2, f}$, D. Viehland ${ }^{2, g}$ \\ ${ }^{1}$ Normandie Univ, France ; UCBN, GREYC, F-14032 Caen, France; CNRS, UMR 6072, F-14032 \\ Caen, France \\ ${ }^{2}$ Materials Science and Engineering, Virginia Tech., Blacksburg, VA 24061, USA \\ ${ }^{3}$ U.S. Naval Research Laboratory, Washington, D.C. 20375

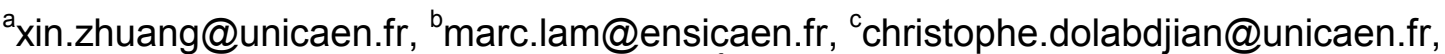 \\ dyaojin@vt.edu, ${ }^{\text {ep }}$ eter.finkel@nrl.navy.mil, ${ }^{f}$ jiefang@mse.vt.edu, ${ }^{9}$ viehland@mse.vt.edu
}

Keywords: magnetoelectric, magnetic noise, modulation

The low frequency performances of a layer-bonded Magneto(elasto)Electric (ME) magnetic sensor have been investigated for an in-plane excitation. This technique is based on the strain modulation at the first longitudinal mechanical resonance of the ME magnetic sensor, where the magnetoelastic properties can be controlled by an applied low frequency magnetic field. Its sensitivity can be derived from the elastic coupling equations with nonlinear factors for the excitation [1]. The equivalent magnetic noise results mainly from mechanical instabilities, due to the arbitrary rotation of elastic domain walls [2]. This modulation relies on the change of either the direct or the converse transfer function as a function of the low frequency magnetic field. Because of the symmetrical magnetic and electric behaviors of the magnetoelectric composite, the direct and converse ME modulations are similar in performances. The magnetoelastic coupling parameter is controlled by the external low frequency magnetic field and dominated by the piezomagnetic coefficient [3]. The mechanical impedance of the composites can also vary as a function of external mechanical stress (this stress can result from the applied magnetic field). However, this is not regarded as a dominant effect for low frequency field sensing.

By using the strain modulation based on the direct or converse ME effects, the layer-bonded ME multilayer exhibits a measured limit of the order of 10 to $100 \mathrm{pT} / \sqrt{\mathrm{Hz}}$ at $1 \mathrm{~Hz}$, as well as a direct current (DC) detection capability under an excitation close to the resonant frequency. The results for both the direct and converse modulation were investigated and compared with the help of an equivalent circuit modeling.

\section{Introduction}

The intermediated strain can convert a magnetic field to an electric output signal in a magnetostrictive-piezoelectric layered composite via three parameters: the magnetoelastic coupling, the piezoelastic coupling and the mechanical impedance. These three parameters are dominated respectively by the magnetostrictive coefficient, the piezoelectric coefficient and the mean flexibility of material in the composite. Focusing on these three parameters, many investigations on the ME enhancement have been carried out by choosing the correct material or by adjusting the ratio between the two phases in the composite [4]. Thereafter, the noise performance of ME laminates has been studied for applications as a magnetic sensor. In the last several years, the intrinsic noise sources for both the composite and the amplifier circuit have been mathematically modeled and experimentally characterized. The passively sensed signal can be amplified by either a voltage or a charge method. Furthermore, the noise contributions from the detection electronics were also integrated in the noise performance analysis [5]. According to these studies, dielectric dissipation in the piezoelectric phase 
is the main contribution to the noise floor for low-frequency magnetic field sensing even though the equivalent current noise source from the electronics induce fluctuations in the output signal of the low-frequency charge detection as well [6].

The amplitude ratio between the output voltage and the magnetic field to be sensed is defined as the ME coefficient for the passive detection method. This coefficient can be modulated by a low frequency magnetic field [7]. Thus, the modulation method is realized for low frequency magnetic field sensing. In practice, the process is achieved by measuring the amplitude of a high frequency carrier, which varies as a function of the low frequency magnetic field. This carrier can be actively driven by either a magnetic or an electric sinusoidal oscillation [8]. In our experiments, a home-made coil serves to generate the high frequency magnetic excitation, whereas, the voltage applied across the interdigital electrodes can excite the sensor via an electric field.

\section{Theory and experimental results}

The modulation detection is based on the strain-coupled direct and converse ME effects produced by a low frequency magnetic field which can modulate the magnetoelastic coupling. Thus, by applying a high frequency excitation oscillation, an amplitude modulation can be achieved by the low frequency magnetic field. A sharp increase of the magnetoelectric coupling has been observed at the mechanical resonant frequency of the ME laminates. Therefore, modulation techniques can be applied at this frequency for sensing low frequency magnetic fields. A layered magnetoelectric laminate consists of a piezoelectric layer, sandwiched between two magnetostrictive layers. The piezoelectric layer is made of five macro-piezoelectric fibers $\left(40 \times 2 \times 0.2 \mathrm{~mm}^{3}\right.$ for each fiber) with a main polarization in the length direction. Each magnetostrictive layer consists of three bi-dimensional Metglas sheets stacked together. The main magnetostriction direction appears along its length direction. Additionally, two pairs of interdigital electrodes serve to measure the generated electronic signal from the piezoelectric layer. These electrodes are deposited on 'Kapton' layers, which have good permittivity and elastic behaviors, and were glued to the top and bottom surfaces of the piezoelectric layer.

Two modulation techniques have been investigated with the help of a pair of constitutive equations at first. The direct and converse ME couplings were theoretically demonstrated, with the equations given in (1) below. From these two equations, the ME coefficients can be derived with the help of nonlinearity factors. These two nonlinear ME coefficients are given in (2) and they represent the magnetic field sensitivity for direct and converse magnetoelectric effects. So, we know that the sensitive parameter to magnetic field is the magnetoelastic coupling in both cases. However, the coefficients in these two coupling parameters are respectively the efficient magnetostrictive and piezomagnetic coefficients [10].

$$
\begin{gathered}
\left\{\begin{array}{l}
\alpha_{M E}=\omega \frac{\partial Q}{\partial H}=\omega_{e x}\left(\varphi_{m} \varphi_{p} C_{m e c h}\right) \\
\alpha_{E M}=\omega \frac{\partial \psi}{\partial E}=\omega_{e x}\left(\varphi_{m} \varphi_{p} C_{m e c h}\right)
\end{array}\right. \\
\left\{\begin{array}{l}
\alpha_{M E_{-} M / E}^{N L}=\frac{\omega_{e x}}{\mu_{0} l} \eta_{1} \varphi_{m} \varphi_{p} C_{m e c h}^{E H} \\
\alpha_{M E_{-} E / M}^{N L}=\frac{\omega_{e x}}{\mu_{0} l}\left(2 \varphi_{m} \tau_{1}\right) \varphi_{m} \varphi_{p} C_{m e c h}^{E H}
\end{array}\right.
\end{gathered}
$$

The mechanism of the direct ME modulation (noted as $\mathbf{M} / \mathbf{E}$ ) is based on the detection of the modulated strain around the mechanical resonance. This magnetic-induced strain is converted into an electric signal via the stacked piezoelectric phases across interdigital electrodes. However, the converse ME modulation (noted as $\mathbf{E} / \mathbf{M}$ ) depends on the detection of a modulated magnetization by a 
pick-up coil. This magnetization is induced by the stress from the piezoelectric layer. For the first case, the excitation and detection parts are respectively the excitation coil and the piezoelectric layer. However, in the latter case the piezoelectric layer and pick-up coil serve respectively as an excitation input and for detection. Experimental configurations of the modulation based on either the direct or the converse ME modulation is given respectively in Fig. 1 (a) and (b).

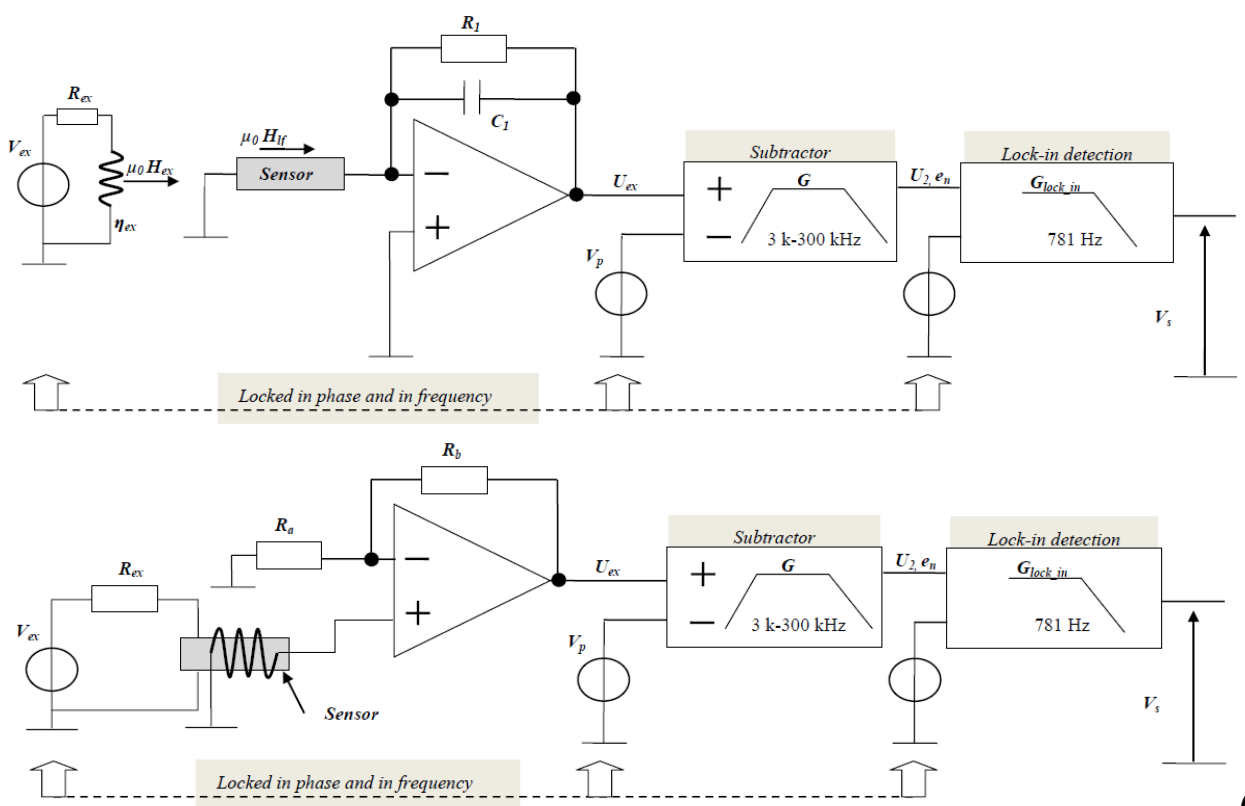

(a)

(b)

Fig. 1: Experimental configurations for (a) direct ME and (b) converse ME modulations.

Shown in Fig. 1 (a) is the charge amplifier with a resistance $R_{l}$ and a capacitor $C_{l}$ as feed-back. The transfer function for the M/E mode is given by $T_{r} \approx \frac{\alpha_{M E}^{N L} M / E}{C_{1}}$. In Fig. 1 (b) the transfer function for the $\mathbf{E} / \mathbf{M}$ mode is $T_{r_{-} E / M}=\alpha_{M E_{-} E / M}^{N L} \times \frac{R_{b}}{R_{a}}$ with the voltage gain of the amplifier $\frac{R_{b}}{R_{a}}$. A band-pass filter, with a gain $G$ and a bandwidth from $3 \mathrm{kHz}$ to $300 \mathrm{kHz}$, was used after both the charge and voltage amplifier as a subtractor. This subtraction serves as a nulling system in order not to exceed the reserve of the demodulation and measuring instruments [10].

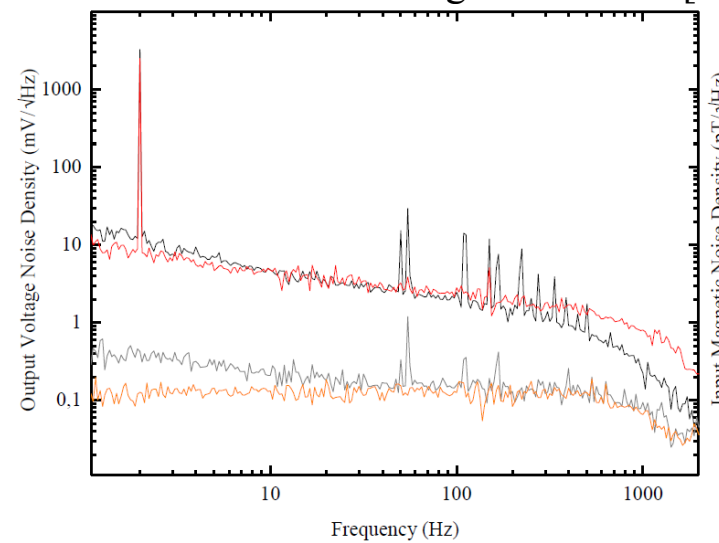

(a)

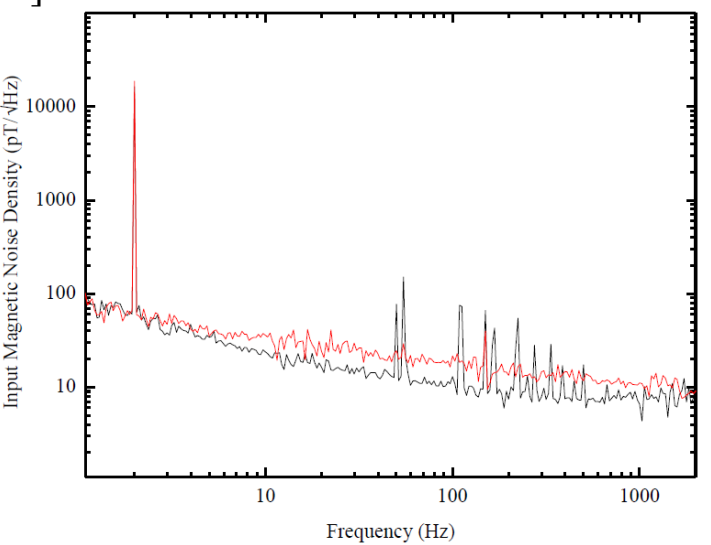

(b)

Fig. 2: (a) The output voltage noise spectral density and (b) The equivalent magnetic noise spectral density as a function of frequency for (black) direct and (red) converse ME modulations.

The output voltage noise spectral density and equivalent magnetic noise spectral density are shown in Fig. 2 (a) and (b). The transfer function curves of $\mathbf{M} / \mathbf{E}$ and $\mathbf{E} / \mathbf{M}$ modes show respectively a nonlinear charge coefficient of $1.2(\mu \mathrm{C} / \mathrm{T}) /(\mathrm{A} / \mathrm{m})$ and a nonlinear voltage coefficient of $1(\mathrm{~V} / \mathrm{T}) /(\mathrm{V} / \mathrm{m})$. The gray and orange curves in Fig. 2 (a) are the measured noise floor from 
respectively the excitation scheme and the lock-in detection. These two noise contributions are much lower than the noise contribution from the sensor. The equivalent magnetic noise floors are shown in Fig. 2 (b). The two modulation techniques have a similar noise level, especially at low frequencies. This is due to the similar modulation mechanisms for these two modulations.

\section{Summary}

A strain-layered magnetoelectric composite can serve as a magnetic sensor for passive detection via the strain coupling. Parametric sensing can be realized by means of the modulations induced by a low frequency magnetic field, which result from direct and converse magnetoelectric changes. The modulation mechanisms are similar for the two methods and thus, their equivalent magnetic noise levels are close to each other. A better performance for low frequency magnetic field sensing will require higher magnetoelastic nonlinearity factors and lower mechanical instabilities as expected from the calculations and current measurements.

\section{Acknowledgement}

The authors would like to thank ONR global for support of this work.

\section{References}

[1] M. D. Mermelstein and A. Dandridge, Dynamic sensitivity and thermal noise analysis of a magnetoelastic morphous metal low-frequency magnetometer, Appl. Phys. Lett., 51 (1987) 1640.

[2] Z. Wang, R. Zhang, E. Sun and W. Cao, Contribution of domain wall motion to complex electromechanical coefficient of $0.62 \mathrm{~Pb}(\mathrm{Mg} 1 / 3 \mathrm{Nb} 2 / 3) \mathrm{O} 3-0.38 \mathrm{PbTiO} 3$ crystals, J. Appl. Phys., 107 (2010) 014110.

[3] D. C. Jiles, Theory of the magnetomechanical effect, J. Phys. D: Appl. Phys, 28 (1995) 1537.

[4] M. I. Bichurin, V. M. Petrov and G. Srinivasan, Theory of low-frequency magnetoelectric coupling in magnetostrictive-piezoelectric bilayers, Phys. Rev. B, 68 (2003) 054402.

[5] X. Zhuang, M. Lam Chok Sing, C. Cordier, S. Saez, C. Dolabdjian, J. Das, J. Gao, J Li and D. Viehland, Analysis of noise in magnetoelectric thin-layer composite used as magnetic sensor, IEEE Sensors J., 11 (2011) 2183.

[6] Z. Xing, J. Zhai, S. Dong, J. Li, D. Viehland and W G. Odendaal, Modeling and detection of quasi-static nanotesla magnetic field variations using magnetoelectric laminate sensors, Meas. Sci. Technol., 19 (2008) 015206.

[7] X. Zhuang, C. Cordier, S. Saez, M. Lam Chok Sing, C. Dolabdjian, J. Gao, J Li and D. Viehland, Theoretical analysis of the intrinsic magnetic noise spectral density of magnetostrictive-piezoelectric laminated composites, J. Appl. Phys., 109 (2011) 124512.

[8] J. G. Wan, J.-M. Liu and G. H. Wang, Electric-field-induced magnetization in $\mathrm{Pb}(\mathrm{Zr}, \mathrm{Ti}) \mathrm{O}_{3} /$ Terfenol-D composite structures, Appl. Phys. Lett., 88 (2006) 182502.

[9] G. Liu, Y. Zhang, P. Ci and S. Dong, Enhanced electrical manipulation of magnetic susceptibility in ferromagnetic amorphous alloy and piezoelectric bimorph heterostructure, J. Appl. Phys., 114 (2013) 064107.

[10]X. Zhuang, M. Lam Chok Sing, C. Dolabdjian, Y. Wang, P. Finkel, J. Li and D. Viehland, Mechanical noise limits of a strain coupled magneto(elasto)electric sensor operating under a magnetic or an electric field modulation, IEEE Sensors J., submitted June 2014. 